\title{
Meta
}

Journal des traducteurs

Translators' Journal

\section{Haskell, Ann Sullivan, " The Lexicon of the Sports and Racing Car Enthusiast ", PADS (Publication of the American Dialect Society), Number 42, November 1964, p. 1-10.}

\section{Blake T. Hanna}

Volume 12, numéro 2, juin 1967

URI : https://id.erudit.org/iderudit/017761ar

DOI : https://doi.org/10.7202/017761ar

Aller au sommaire du numéro

Éditeur(s)

Les Presses de l'Université de Montréal

ISSN

0026-0452 (imprimé)

1492-1421 (numérique)

Découvrir la revue

Citer ce compte rendu

Hanna, B. T. (1967). Compte rendu de [Haskell, Ann Sullivan, " The Lexicon of the Sports and Racing Car Enthusiast ", PADS (Publication of the American Dialect Society), Number 42, November 1964, p. 1-10.] Meta, 12(2), 58-59.

https://doi.org/10.7202/017761ar d'utilisation que vous pouvez consulter en ligne.

https://apropos.erudit.org/fr/usagers/politique-dutilisation/ 
ce qu'il faut savoir pour parler et écrire correctement. La seconde analyse et décrit la structure du français contemporain. Il n'y est pas question de règles, mais de mécanismes que l'on démonte devant nous.

Ces mécanismes, le plus souvent nous les utilisons inconsciemment. Aussi le profane qui parcourt ce livre a-t-il le sentiment qu'il n'y apprend rien. En un sens e'est vrai. Nous n'avons pas besoin, si le français est notre langue maternelle, qu'on nous dise que le se place avant lui dans «je le lui donne ». Nous le savons sans l'avoir jamais appris. Mais le but de la grammaire structurale n'est pas de nous apporter des préceptes, il est de nous faire comprendre que la langue est un système et que les pièces du système sont agencées d'une certaine façon.

Ce nouvel éclairage est très instructif pour celui qui connâ̂t assez bien la grammaire normative. Chemin faisant, l'occasion lui est donnée d'approfondir certaines choses. Peut-être ne s'était-il jamais rendu compte que le français oppose l'animé à l'inanimé: duquel peut se dire des choses et des personnes, de qui ne s'emploie que des personnes. Et il arrive que cette opposition l'emporte sur celle de l'objet et du sujet: $Q u i$ voyez-vous? Que voyez-vous?

Voici deux autres exemples de cette façon d'envisager les faits de langue. L'énoncé et les segments qui le constituent véhiculent, en principe, une certaine quantité d'information. Il arrive que cette information soit redondante. Dans ce eas, le segment qui la porte est utilisé stylistiquement. Ainsi s'explique l'emploi de l'article dans « la Louise » et du démonstratif dans « ce type-là », qui ne s'oppose pas à « ce type-ci ».

Quand l'adjectif est antéposé, il est uni plus étroitement au nom qu'il qualifie. On le savait déjà, mais on en fait la démonstration en constatant que si «le velours rouge » peut devenir « le velours» (dont on sait qu'il est rouge), par contre 《le grand homme» ne peut pas être réduit à «l'homme». Si cette réduction est possible dans le cas de « une vive douleur », c'est parce que vive peut se placer aussi bien après qu'avant douleur.

Les points de vue auxquels se place l'auteur, la terminologie qu'il emploie, montrent bien que son livre a été écrit pour les linguistes. De toute évidence, il ne s'adresse pas aux écoliers qui ont à repasser les pluriels irréguliers. Cependant on peut en conseiller la lecture à tous ceux qui, sans être spécialistes, s'intéressent aux questions de langue. Ils verront que si l'auteur est souvent difficile à suivre, il mène toujours quelque part, et ils lui sauront gré de leur avoir fait découvrir de nouveaux horizons.

Jean Darbeinnet

Haskell, Ann Sullivan, «The Lexicon of the Sports and Racing Car Enthusiast 》, PADS (Publication of the American Dialect Society), Number 42, November 1964, p. 1-10.

The car enthusiast's lexicon published in this, the latest, issue of $P A D S$ will keep many a translator from coming unglued ${ }^{1}$ when faced with the com-

1. «Come unglued, v. phr. To blow up or disintegrate », PADS, Number 24, p. 3. 
plexities of drag racing terms. While hardly exhaustive - no such glossary ever is - the article has culled from Road and Track, Sports Car Graphic and ten other periodicals catering to automobile enthusiasts a selection of terms in current use among specialists in the field. Thus, a D.O.H.C. (double overhead camshaft) is distinguished from a S.O.C. (single overhead camshaft), while a spyder turns out to be, not an arachnid with four pairs of hairy legs, but rather a racing ear with such equipment as headlights, brake lights, etc., necessary to qualify it legally to be driven on a public highway.

Published by the University of Alabama Press, $P A D S$ oceasionally publishes articles of interest to the translator. Thus Number 26 (November 1956) is devoted to a penetrating report entitled: «Bilingualism in the Americas», by Einar Haugen, accompanied by a 31-page bibliography, while Number 24 (November 1955) contains an exhaustive, 200-page article on underworld slang entitled: «Whiz Mob», by David W. Maurer. The terms contained in the latter are indexed in Number 31 (April 1959)².

BuAKE T. HaNNA

\section{"Catalogue des livres canadiens en librairie"}

Dans son dernier rapport annuel, le bibliothécaire fédéral a annoncé le projet de publication d'un ouvrage de référence qui sera bien accueilli des bibliographes. Il s'agit d'une publication annuelle intitulée Canadian Books in Print, qui viendra rejoindre dans les salles de référence des bibliothèques le British Books in Print, le Books in Print (U.S.A.), la Bibliographie de la France et autres répertoires de publications. Il semble (le bibliothécaire ayant «oublié » de le préciser) que le Canadian Books in Print aura un équivalent, ou du moins un titre, français: le Catalogue des livres canadiens en librairie. Il sera établi avec l'aide d'un ordinateur et publié sous les auspices des associations d'éditeurs canadiens.

PaUl A. Horguelin

\section{Centre de documentation technique}

Le Centre de documentation technique de Montréal, animé par un ingénieur, M. Édouard Girollet, a pour but essentiel d'informer les milieux québécois intéressés sur les techniques, les matériels et les réalisations françaises, et réciproquement. Il ne s'agit pas d'un organisme statique se bornant à mettre à la disposition de ses visiteurs des collections de livres et de revues.

Le Centre s'efforcera d'apporter des réponses aussi rapides et complètes que possible aux problèmes précis préoccupant ses correspondants. Il jouera un rôle actif de prospection et d'information auprès des sociétés, organismes, ministères québécois, et mettra à leur disposition une documentation technique adaptée à leurs besoins.

2. PADS, Number 31, p. 14-30. 